5. Es bestätigt sich, dass das gewöhnliche Wasser grössere Urinmengen zur Folge hat, als das Thermalbad. Wahrscheinlich liegt die Differenz bier in respirationsseitig vermehrt ausgeschiedenem Wasserdampf. -

6. In kühlen Bädern steigt die Achseltemperalur des Badenden häufig, wenn auch nicht ausuahmslos, sinkt ausnahmslos in der Nachbadeperiode, zeigt aber nach dem Thermalbade eine ungewöhnliche Höhe. -

7. Das $28^{\circ}$ warme Thermalbad und das $6^{\circ}$ warme Sitzbad zeigen mehr Aehnlichkeit der Einwirkung, als das $28^{\circ}$ warme gewöhnliche Wasser. Die Aehnlichkeit besteht bei Beiden in Röthung der Haut und Vortäuschung einer löheren Temperatur.

8. Das warme, gewöhuliche Bad ist ein gutes, die Diurese steigerndes Mittel, wirksamer als die anderen, hier untersuchten. -

9. Das Thermalbad ist ein gutes, die Respirationsenergie stejgerndes Mittel, wirksamer als die anderen, lier untersuchten. -

10. Alle Bäder bewirken eine Zeit lang durch Puls - und Respirationsverminderung, so wie durch Abkühlung (Ausnahme Thermalbald), Ermüdung und Schläfrigkeit, in welcher Beziehung das $35^{\circ}$ warme Sitzbad am wirksamsten ist.

11. Es giebt eine balneosensible, balneomusculäre, balneothermische, balneolylische und hautröthende Einwirkung einiger Bäder, Einwirkungen, welche denen electrischer Ströme analog sind.

\title{
VIII.
}

\section{Ueber den inneren Gebrauch von Atropinum sulphuricum bei profusen Schweissen, namentlich bei Nachtschweissen der Plitlisiker.}

\author{
Von Ober-Stabsarzt Dr. Fräntzel, \\ Docenten an der Universiät und dirigirendem arzte an der Charité.
}

Eine Notiz in der Philadelphia Medic. Times (1872. No.51, cf. Centralblatt 1873. S. 96), wonach Dr. J. C. Wilson auf Veranlassung von Da Costa im Pensylvania-Hospital vier Leuten mit vorgeschrittener Lungenschwindsucht, welche an profusen Nacht- 
schweissen litten, täglich innerlich $\frac{1}{60}$ Gran Atropinum sulphuricum gereicht und dadurch eine völlige Beseitigung der Nachtschweisse erzielt hat, bewog mich, weitere Versuche in Bezug auf die Wirkung des Atropins gegen profuse Schweisse und namentlich gegen die Nachtschweisse der Phthisiker auf meiner Abtheilung in der Charité und bei den unter meiner Behandlung befindlichen internen Kranken des Augusta-Hospitals anzustellen. Obgleich ich an die Darreichung des Mittels mit grossem Unglauben heranging, so hielt ich doch sorgfältige Beobachtungen in Bezug auf die etwaige Richtigkeit der Angaben von Wilson für geboten, weil die ejgentlich völlige Erfolglosigkeit aller anderen Mittel gegen die in Rede stehenden Nachtschweisse und die rasch zunehmende Erschöpfung der Kranken durch dieselben ganz abgesehen von der grossen Belästigung, welche die Patienten dadurch erfahren, in jedem Arzt das Verlangen nach einem wirksamen Medicament erwecken muss.

Nachdem ich nunmehr in 75 sorgfältig beobachteten Fällen vom Atropinum sulphuricum Gebrauch gemacht habe, glaube ich auf Grund dieser Beobachtungen den Fachgenossen das Mittel gegen profuse Schweisse der Phthisiker sowie gegen lästige Schweisse bei anderen Krankheiten $\%$. B. beim Rheumatismus articulorum acutus und bei Reconvalescenten von Trichinosis entschieden empfehlen zu können. Der Erfolg ist kein unfehlbarer, auch tritt nieht immer eine völlige Sistirung der Schweisse, aber doch in der überwiegenden Mehrzahl der Fälle eine sehr erhebliche Besserung ein, so dass die Kranken wenigstens aufhören sich belästigt und erschöpft zu fühlen und der sichtbare Collapsus nicht weiter fortschreitet.

Unter meinen 75 Fällen befanden sich 15 Individuen mit mehr oder weniger frischen käsigen Pneumonien, die alle mehr oder weniger fieberten und bei denen Nachtschweisse aufgetreten waren, 48 mit ausgesprochener Phthisis pulm., von denen 42 fieberten, 8 Fälle von Rheumatismus articulorum acutus mit erheblichem Fieber, zwei von Endocarditis ulcerosa und zwei Fälle von Trichinose. Unter den ersten 15 Kranken wurden bei sechs die Schweisse völlig beseitigt, bei sieben sehr erheblich vermindert, bei zweien nicht verändert. Bei den 48 Phthisikern blieb 5 Mal das Mittel ohne jeden Erfolg, 21 Mal liessen die Schweisse sehr beträchtlich nach, 22 Mal schwauden sie ganz. Dabei befanden sich unter den Kranken, welche erfolglos mit Atropin behandelt wurden, mehrere kurz ante 
finem vitae, was möglicherweise von Einfluss auf den Erfolg gewesen ist. Beim Rheumatismus articulorum acutus war das Atropin 5 Mal von dauernd günstiger Wirkung, $2 \mathrm{Mal}$ bedingte es einen wesentlichen Nachlass der Schweisse, $1 \mathrm{Mal}$ war es so gut wie nutzlos. Unter den erwähnten zwei Fällen von Endocarditis nutzte es einmal, das andere Mal absolut nicht. Die Schweisse bei zwei Reconvalescentinnen von Trichineninvasion wurden nach Darreichung des Atropin sofort und dauernd beseitigt.

Letztere Erfolge und der prompte Effect, den ich durch Atropin bei meinen ersten Versuchen gegen die profusen Schweisse beim Rheumatismus articulorum acutus erzielte, flössten mir ein wirkliches Vertrauen zu dem Mittel ein, das sich nachher nur befestigt hat.

$D$ ie bejden Trichinenerkrankungen babe ich in der Privatpraxis beobachtet. Die Details der Krankheit dürften hier füglich zu übergehen sein. Nur soviel sei erwăhnt, dass in beiden Fällen nach Ablauf des acuten Stadiums der Krankheit und des hectischen Fiebers, welches wie gewöhnlich in schwereren derartigen Fällen den Uebergang aus der Febris continua zur Reconvalescenz bildete, ohne jede Spur von Temperafurerhöhung profuse Schweisse persistirten, welche gegen Abend begannen und bis in die Nacht hineindauerten. Das eine Mal wurde 2 Stunden vor dem gewöbnlichen Beginne des Schweisses 0,001 Atrop. sulphur. in Pillenform 5 Tage hinter einander, das zweite Mal nur an 3 auf einander folgenden Tagen gegeben, worauf die Schweisse vom ersten Abend nach Anwendung des Mittels an für immer verschwanden.

Unter den 8 Kranken mit Polyarthritis rheumatica war bei dem ersten Kranken der Erfolg ein wirklich überraschender.

Der 32jährige Patient war bereits à Tage krank, fast alle grösseren Gelenke der Ober- und Unterextremitäten waren mebr oder weniger stark afficirt, der Kranke vollkommen mit Sudaminibus aberdeckt und bei meiner Visite um $10 \mathrm{Jbr}$ Morgens wie in Schweiss gebadet. Es wurde sofort 0,001 Atrop. sulphur. gegeben und schon eine Viertelstunde später machte sich ein Nachlass im Schweiss bemerkbar, 2 Stunden darauf war der Schweiss ganz verschwunden. Nachts kehrte er wieder und hörte Vormittags nach Darreichung einer solchen Atropinpille wieder anf. Vou nua an wurde Abends und Morgens je eine solche Dosis Atropin gereicht and auf diese Weise die Wiederkehr der Schweisse ganz verhütet, während das Fieber erst 14 Tage nach der Aufnahme des Patienten in das Krankenhaus völlig verschwunden war.

In einem anderen Falle von acutem Gelenkrheumatismus handelte es sich um einen 5ljälrigen Tischler, welcher in den letzten Jahren wiederholt an Gelenk- 
rheumatismus gelitten hatte und an 25. Januar 1873 von Neuem erkrankt war. Am 27. Januar suchte er Hülfe im Augustahospital: Beide Kniegelenke und das rechte Ellbogengelenk waren ziemlich stark geröthet und geschwollen, gegen Druck sehr stark, spontan mässig empfindlich. Keine Herzaffection, profuse Schweisse. A. T. 39,5, P. 104, K, 30 . Ordination: Vesicatore über die schmerzhaften Gelenke nach der Davies'scben Methode, subcutane Morphiuminjection. Am 28. Morg. T. $38,7^{0}$ C., P. 92, R. 24. Profuser Schweiss, keine neue Gelenkaffection, die Schmerzhaftigkeit der gestern erkrankten Gelenke viel geringer. Die durch die Vesicatore bewirkten Blasen werden mit der Scheere entfernt, die wunden Flächen bis zum Abend mit Cataplasmen bedeckt, dann mit Ungt. simpl. verbunden. Abds. T. $38,5^{\circ}$, P. 90 , R. 24 . Ordination: 0,001 Atrop. sulphur. Abends 8 Uhr.

Gegen 9 Uhr liess der Schweiss nach und war gegen 10 Uhr völlig verschwunden. In der ganzen Nacht kein Schweiss.

29. Jan. Morg. T. 38,2, P. 88, R. 24,

Abds. T. 38,1, P. 88 , R. 24 .

In Bezug auf die Gelenke keine wesentliche Veränderung. Am Tage kein Schweiss, erst gegen Abend leichte Transspiration. Abends 0,001 Atrop. sulph.

30. Jan. Nachts kein Schweiss.

Morg. T. 38,0, P. 84, R. 22,

Abds. T. 38,1 , P. 86, R. 22.

Nachlass in den rheumatischen Affectionen, im Laufe des Tiges mässiger Scbweiss. Abends 0,002 Atrop. sulphur.

31. Jan. Nachts kein Schweiss, auch in Laule des Tages Schweiss nicht wiedergekehrt. Während das Fieber in den nächsten Tagen noch in geringer Intensität fortbestand, und die Gelenkschwellungen und Schmerzen nur langsam sich minderten, gelang es durch 0,002 Atrop. sulphur. Abends gereicbt die Schweisse völlig zu beseitigen.

Am 4. Februar wurde das Atropin ausgesetzt, in der Nacht und am folgenden Tage schwitzte der Kranke wieder ziemlich stark. Am 5. Febr. Abends nahm er von Nenem 0 002 Atropin. Die Scbwejsse hörten wieder schon mit Beginn der Nacbt auf und blieben bei regelmässigem Gebrauch dieser Dosis am Abend verschwunden, bis sie am 8 . Abends, als Patient bereits fieberlos geworden war und das Atropin wieder ausgesetzt wurde, von Neuem wenngleich mit geringerer Heftigkeit auftraten. Am 9. Abends wurde wieder Atropin gegeben und zwar mit promptem Erfolge. Jetzt wurde das Mittel noch 8 Tage fortgesetzt, dann blieben die Schweisse für immer fort. Am 7. März wurde der Kranke geheilt entlassen.

Derartige Beobachtungen, dass nach Aussetzen des Atropins die Schweisse sofort wiederkehrten, um bei Wiederaufnahme des Mittels 2u verschwinden, habe ich 8 Mal bei Phthisikern registrirt und balte dieselben für geeignet, stringent $\mathrm{zu}$ beweisen, dass in der That dem Atropin dieser Einfluss auf die Schweisse zuzuschreiben ist. Die Kranken fühlten sich durch die enorme Erleichterung, welche ihnen auf diese Weise zu Theil wurde, sehr glücklich und bauten mit 
grosser Sicherheit auf die Wirkung desselben. Auch das intelligente Pflegepersonal des Augusta-Hospitals war so bald von dem in der überwiegenden Mehrzahl der Fälle eintretenden Erfolg überzeugt, dass sie das Atropin, welches ich stets in Pillenform in folgender Formel:

Atropin. sulphur. 0,006

Extr. Gentian. q. s. ut f. pilul. No. X Consp. semine Lycop. entweder in einer oder zwei derartigen Pillen reichte, als Schwitzpillen bezeichneten.

Grössere Dosen als 0,0012 Atropin habe ich nie verabfolgt, weil ich eine intensivere Intoxication scheute. Allerdings habe jch von den von mir verabfolgten Dosen zu 0,0006 und 0,0012 niemals Nacbtheile, wohl aber doch leichte Intoxicationserscheinungen bemerkt. In nicht wenigen Fällen fühlten die Kranken nach dem Einnehmen ein Kratzen im Halse, das aber immer ziemlich rasch d. h. nach ein oder zwei Stunden verschwand, die Pupillen reagirten nicht selten nach dem Einnehmen etwas träge und waren mänchmal auch weiter wie vorher; zuweilen wurde Flimmern vor den Augen beobachtet. In 4 Fällen musste das Mittel wegen profuser Durchfälle ausgesetzt werden; dass diese mit dem Gebrauch des Atropins in Verbindung standen, ging daraus hervor, dass mit dem Weglassen desselben die Durchfälle schwanden und wieder auftraten, sobald von Neuem Atropin gegeben wurde. Irgend welche andere Nachtheile habe ich nie gesehen, während der Volksglaube ja die Unterdrückung jedes Schweisses für nachtheilig hält. Die kritischen Schweisse der Pneumoniker oder Recurrenskranken babe ich nie zu beseitigen versucht, weil ich hierdurch wohl mit Recht fürchtete, eine Störung der eingeleiteten Krise zu bewirken.

Man darf demnach wohl als empirisch feststehend ansehen, dass die profusen und lästigen Schweisse, welche die Lungenschwindsucht, die käsige Pneumonie, den Gelenkrheumatismus und die Reconvalescenz der Trichinose und andere Krankheiten häufig begleiten, durch regelmässigen inneren Gebrauch von 0,001 bis 0,002 Atrop. sulphur. in der überwiegenden Mehrzahl der Fälle entweder ganz beseitigt oder wenigstens erheblich vermindert werden. Fragt man aber, in welcher Weise die Wirkung zu Stande kommt, so ist darauf äusserst schwer zu antworten. Denn zunächst ist der physiologische Vorgang der Schweisssecretion noch nicht 
zweifellos festgestellt, zweitens bedarf er einer Erklärung für das Zustandekommen der Schweisse bei den oben erwähnten Krankheiten, drittens aber sind unsere Kenntnisse von der Wirkung des Atropins trotz vielfacher sehr verdienstvoller Arbeiten hierüber nicht so vollständige, um die geschilderte Wirkung des Mittels ohne Weiteres erklären zu können.

Ich bin geneigt anzunehmen, dass die profusen Schweisse in den erwähnten Krankheiten ebenso wie die Schweisse, welche wir bei heruntergekommenen schlecht genährten Individuen nach nur geringen Körperanstrengungen ohne direct nachweisbare Krankheiten auftreten sehen, durch eine Erschlaffung der Wandungen der peripheren zu den Schweissdrüsen gebörigen Gefässe bedingt sind. Atropin bedingt nun, wie aus den Versuchen von Meuriot, Fleming, Jones, Hayden sowie denen von Brown-Séquardan der Schwimmhaut von Fröschen hervorgeht, eine stapke Verengerung des kleinsten Arterien und damit ein Aufhören des Schweisses. Die ersten durch Belladonna und seine Präparate bedingten Intoxicationserscheinungen, die Trockenheit der Schleimhaut des Mundes und des Rachens (cf. Nothnag el, Arzneimittellehre. Berlin 1870. S. 55 u.ff.) und der Haut, welche letztere sich bei hohen Graden der Atropinwirkung rollständig pergamentartig anfühlt, während zugleich die Feinheit des Tastgefühls abnimmt, das Aufhören der Secretion des Speichels, der Thränen und des Nasenschleims sind offenbar in gleicher Weise zn erklären. 